\title{
Preferencia por contenidos científicos de física o de biología en Educación Primaria: un análisis clúster
}

\author{
Radu Bogdan Toma \\ Departamento de Didácticas Específicas. Facultad de Educación. Universidad de Burgos. Burgos. España. \\ rbtoma@ubu.es \\ ORCID: bttps:// orcid.org/0000-0003-4846-7323 \\ Jesús Ángel Meneses Villagrá \\ Departamento de Didácticas Especificas. Facultad de Educación. Universidad de Burgos. Burgos. España. \\ meneses@ubu.es \\ ORCID: https:/ / orcid.org/0000-0003-4839-0418
}

[Recibido: 8 Junio 2018. Revisado: 9 Octubre 2018. Aceptado: 22 Octubre 2018]

\begin{abstract}
Resumen: Las estadísticas de matriculación en carreras de ciencia, tecnología, ingeniería y matemática (STEM) evidencian una gran infrarrepresentación de las mujeres en la mayoría de estas disciplinas, especialmente en física e ingeniería. En este estudio se analiza el interés de niñas y niños de Educación Primaria $(n=733)$ por el contenido de disciplinas científicas de física y de biología utilizando un método de análisis cuantitativo basado en técnicas de agrupamiento por conglomerados K-Medias, analizándose las diferencias en las actitudes de los estudiantes hacia la ciencia escolar en función de su interés por estas disciplinas científicas. Los resultados revelan la existencia de un perfil actitudinal sesgado sexualmente, caracterizado por un alto interés por contenidos propios de ciencias biológicas y un bajo interés por el aprendizaje de la física por parte de las niñas, y resultados opuestos en el caso de los niños. Además, en comparación con los niños, las niñas han mostrado menos interés por contenidos de física, han sido mayoría en los perfiles actitudinales caracterizados por un bajo interés general por contenidos científicos y han sido minoría en los perfiles caracterizados por un alto interés por ambas disciplinas. Estos resultados parecen mostrar que el interés por disciplinas científicas de física o de biología empieza a forjarse desde niveles elementales del sistema educativo, hecho que podría dar lugar a la posterior segregación de género en las disciplinas STEM. Se discuten las implicaciones educativas y de investigación de estos resultados.
\end{abstract}

Palabras clave: actitudes hacia la ciencia, K-medias, conglomerados, ciencias biológicas, ciencias físicas

\section{Preference for physics or biology scientific content in Elementary Education: a cluster analysis}

\begin{abstract}
Statistics on enrolment in science, technology, engineering and mathematics (STEM) show a large under-representation of women in most of these disciplines. This study analyses the preferences of elementary education girls and boys $(n=733)$ for the content of physics and biology using a quantitative analysis method based on clustering techniques (K-Means) and by analyzing the differences in their attitudes toward school science according to their preferences. The results reveal the existence of a sexually biased attitudinal profile, characterized by a high preference for the content of the biological sciences and a low preference for learning physics among girls, and opposite results in the case of boys. In addition, in comparison with boys, girls showed less interest in physics science content than boys, were majority in the attitudinal profiles characterized by a low overall interest in science content and minority in the profiles characterized by a high interest in both disciplines. These results seem to show that the preference for scientific disciplines in physics or biology are beginning to form from elementary levels of the educational system, which could lead to the subsequent gender segregation existing in STEM disciplines. The educational and research implications of these findings are discussed.
\end{abstract}

Keywords: attitudes towards science, K-mean, cluster analysis, biology, physics

Para citar este artículo: Toma, R. B., Meneses Villagrá, J. A. (2019) Preferencia por contenidos científicos de física o de biología en Educación Primaria: un análisis clúster. Revista Eureka sobre Enseñanza y Divulgación de las Ciencias 16(1), 1104. doi: 10.25267/Rev_Eureka_ensen_divulg_cienc.2019.v16.11.1104 


\section{Introducción}

En 2013 más de la mitad de los títulos de estudios superiores en disciplinas de ciencia, tecnología, ingeniería y matemática (STEM) de los países miembros de la Unión Europea fueron obtenidos por mujeres, sin embargo, apenas un 30\% de los graduados en ingeniería eran mujeres (OECD, 2013). Además, en la etapa de Educación Secundaria, cuyas edades comprenden en los 14 y 18 años de edad, las alumnas son una minoría en asignaturas de física, un patrón generalizado en diversos países como Francia (Rapoport y Thibout 2018), Holanda (Buser, Niederle y Oosterbeek 2014) o Australia (Collins, Kenway y McLeod 2000). En términos de ocupación STEM, las mujeres siguen representando un porcentaje muy inferior en comparación con los hombres (Beede et al. 2011; Deloitte 2016; INE 2017; NSF 2013; UNESCO 2017). España no es una excepción. Si bien el número de mujeres en estudios universitarios de ciencias ha aumentado recientemente, aún persiste la disparidad entre el porcentaje de mujeres y de hombres en algunas disciplinas STEM. Así, apenas un 25.4\% de las matriculaciones en titulaciones universitaria de ingeniería y arquitectura pertenecen a mujeres. Además, apenas un $2.5 \%$ del total de egresados en estudios superiores en física y matemáticas son mujeres (INE 2017; MECD 2016). Por lo tanto, la retención de las mujeres en STEM resulta crítica en la actualidad.

En la literatura existen numerosos estudios que indican que el comportamiento y las elecciones de los estudiantes pueden verse moldeadas por sesgos implícitos derivados de la exposición a estereotipos culturales generalizados que conciben la Ciencia como una profesión masculinizada (Dasgupta 2011) y a las mujeres como menos competentes (Eagly y Mladinic 1993), siendo estos estereotipos sostenidos y exhibidos por ambos géneros (Nosek, Banaji y Greenwald 2002). Aunque numerosas investigaciones han confirmado la existencia de sesgos de género en ámbitos familiares y educativos en cuanto a las competencias matemáticas y científicas de las niñas y los niños (Dickhauser y Meyer 2006; Gunderson, Ramirez, Levine y Beilock 2012), aún no resulta claro a partir de qué edad estos estereotipos podrían tener efecto en las vocaciones y el interés de los estudiantes por las disciplinas STEM. Por ello, en este estudio se pretende identificar el interés de las niñas y los niños de Educación Primaria por contenidos de física o de biología y analizar si existen diferencias en sus intereses según la variable sexo y edad. Además, se pretende examinar si existen diferencias en las actitudes hacia la asignatura de Ciencias de la Naturaleza en función de los intereses por sus contenidos. Más específicamente, se pretende descubrir si desde una temprana edad se pueden identificar diferencias en los intereses de los niños y las niñas por disciplinas científicas de física y de biología y, en caso afirmativo, determinar si existen diferencias en las actitudes hacia Ciencias de la Naturaleza en los grupos que tienen mayor interés por una u otra disciplina. Las tres preguntas de investigación que guían el presente estudio son:

P1. ¿Cómo afecta el sexo y el curso escolar a las preferencias por estudiar contenidos científicos de física y de biología?

P2. Las preferencias, ¿simulan patrones de matriculaciones identificadas en etapas secundarias y terciarias?

P3. ¿Existen diferencias en las actitudes de los estudiantes hacia la asignatura de Ciencias de la Naturaleza en función de su preferencia por estudiar contenidos de física o de biología?

De acuerdo con los datos nacionales de matriculación (MECD 2016), se espera identificar un subgrupo de niñas que se caracteriza por un bajo interés por contenidos de física y un alto interés por contenidos propios de las ciencias biológicas, y resultados opuestos en el caso de los niños. Además, en consonancia con investigaciones pasadas que señalan actitudes desfavorables hacia la Ciencia por parte de las niñas, (p. ej.: de Pro Bueno y Pérez Manzano 
2014; Denessen, Vos, Hasselman y Louws 2015; Marbá-Tallada y Márquez Bargalló 2010; Vázquez y Manassero 2008), se espera identificar otro subgrupo de niñas con bajo interés tanto por la física como por la biología. Por último, dado el patrón generalizado reportado en la literatura de actitudes hacia la Ciencia señalando diferencias significativas a favor de los niños (Caleon y Subramaniam 2008; DeWitt y Archer 2015; George 2006; Hacieminoglu 2016), se espera encontrar una mayor proporción de niñas que de niños con bajo interés tanto por la física como por la biología y un mayor número de niños que de niñas con un alto interés tanto por la física como por biología.

\section{Justificación}

En este apartado se adopta una triple-argumentación para justificar por qué los actuales patrones de matriculaciones en ciencias en las etapas secundarias y terciarias del sistema educativo resultan problemáticos; es decir, por qué resulta preocupante la baja presencia de mujeres en determinadas disciplinas STEM como la física o la ingeniería. Así, entre los muchos argumentos que subrayan la necesidad e importancia de aumentar el número de mujeres matriculadas en las disciplinas científicas en las que su presencia es minoritaria, en este trabajo se adopta como fundamentales los siguientes tres: (I) alfabetización científica integral e inclusiva, (II) necesidad de capital humano científicamente alfabetizado, y (III) diversidad en intereses y visiones científico-tecnológicas.

\section{Alfabetización científica integral e inclusiva}

Probablemente uno de los principales argumentos a favor de la inclusión de las niñas en todos los estudios y disciplinas STEM se basa en los derechos humanos (UNESCO 2017). Desde hace décadas, numerosos informes internacionales (AAAS 1993; EC 2013; NRC 2012; OECD y UNESCO 2003; Osborne y Dillon 2008), así como leyes educativas nacionales, como la Ley Orgánica para la Mejora de la Calidad Educativa (LOMCE 2013), vienen resaltando la importancia de alfabetizar científicamente a los estudiantes. La igualdad de género en las disciplinas STEM se postula como importante para garantizar que los niños y las niñas puedan adquirir los beneficios asociados con la alfabetización STEM (UNESCO 2017), algo que no sucederá a menos que se reduzca la gran segregación de género en determinadas disciplinas, como la física. Las escasas matriculaciones en estudios de física por parte de las mujeres resultan aún más preocupantes con la nueva actual ley educativa, en la que los estudiantes configuran su trayectoria académica desde el tercer curso de la etapa Educación Secundaria Obligatoria (ESO) y deben decidir si se matriculan en asignaturas propias de la rama de Ciencias o no. Si desde etapas elementales las niñas no desarrollan actitudes positivas e interés por la física, es muy probable que en Secundaria opten por asignaturas diferentes, por lo que la segregación se produce mucho antes en el sistema educativo, resultando más difícil aún despertar su interés por disciplinas de física en etapas posteriores.

\section{Necesidad de capital humano}

En la actualidad, la falta de mujeres en algunas disciplinas STEM supone una situación problemática tanto para la inclusión educativa como también para la consecución de las demandas del mercado laboral (Beede et al. 2011; EC 2004). La existencia de ciudadanos cualificados en disciplinas STEM es crucial para la capacidad innovadora y el desarrollo tecnológico de los países. En la actualidad, se estima que existe una ausencia de personal cualificado para cubrir los puestos laborales necesarios en STEM (Berkhout, Sattinger, Theeuwes y Volkerink 2012). La falta de personal cualificado combinado con el envejecimiento de la fuerza laboral en STEM suscita un panorama preocupante (Morrell y Parker 2015). Por lo tanto, el aprovechamiento del progreso científico-tecnológico requiere del 
mayor número posible de talentos, suponiendo un gran déficit la falta de inclusión de las mujeres en estas disciplinas (Blickenstaff 2005).

\section{Diversidad en intereses y visiones científico-tecnológicas}

La escasa representación de las niñas en las disciplinas STEM es problemática no solo por cuestiones de inclusión educativa o necesidad de capital humano, sino también debido a las limitaciones que esto supone al desarrollo de las innovaciones científicas. Dado que los hombres y las mujeres pueden albergar intereses y concepciones diferentes, las innovaciones científico-tecnológicas podrían verse obstaculizadas por la falta de diversidad de género en determinadas disciplinas científicas (Sikora y Pokropek 2012). Por ejemplo, en ingeniería, una disciplina predominantemente masculina, se definió la altura y el peso medio de la población estadounidense masculina como la norma y objeto principal de estudio en pruebas de choque (AGARD 1996). La falta de atención a otros segmentos de la población (por ejemplo, las mujeres) durante la fase de diseño de mecanismos de seguridad resultó en daños no intencionales dado que los cinturones de seguridad convencionales no se ajustan a las necesidades de las mujeres embarazadas, por lo que los accidentes automovilísticos son la principal causa de muerte fetal (Weiss, Songer y Fabio 2001). Una mayor presencia de mujeres en estas disciplinas hubiese dado lugar a una visión más holística durante la fase de diseño, siendo las perspectivas diferenciadas aportadas a la Ciencia por las mujeres necesarias para moldear las innovaciones tecnológicas y el propio conocimiento científico de manera significativa (Heybach y Pickup 2017). Las distintas experiencias e inquietudes de las mujeres pueden dar lugar al desarrollo de nuevas preguntas de investigación y perspectivas investigadoras, siendo numerosas las evidencias que manifiestan el impacto de las mujeres en el desarrollo de diferentes disciplinas científicas y que subrayan la necesidad de mayor inclusión de las mujeres en la Ciencia (Bug 2003; Conkey 2003; Schiebinger 2003). Sirva de ejemplo Gendered Innovations (Schiebinger et al. 2011), una iniciativa que proporciona numerosos estudios de casos que ilustran la forma en que las aportaciones de género en la ciencia, la medicina, la ingeniería y en investigación medioambiental condujo a nuevos descubrimientos e innovaciones.

\section{Fundamentación teórica: actitudes hacia la Ciencia}

La literatura sobre actitudes en la Enseñanza de las Ciencias se ve afectada por la falta de claridad y conceptualización del objeto de estudio, es decir, las actitudes (Osborne et al. 2003). Por tanto, la investigación en actitudes hacia la Ciencia se enfrenta a la ausencia de una clara conceptualización de lo que constituye las actitudes hacia la ciencia y también sobre los diferentes términos utilizados para referirse a las actitudes. En relación con la conceptualización de las actitudes, Klopfer (1971) ofreció una visión sobre el concepto objeto de estudio al categorizar las actitudes como un conjunto de comportamientos afectivos hacia la Ciencia, los científicos, la investigación científica, las carreras científicas y hacia las actividades relacionadas con la ciencia en general. Gardner (1975) definió las actitudes hacia la Ciencia como la evaluación favorable o desfavorable de la ciencia. Con respecto a los diferentes términos, Ramsden (1998) llegó a la conclusión de que términos como interés, opiniones, imágenes, creencias y valores se utilizan indistintamente y que sus significados a menudo se superponen. A pesar de los numerosos términos y concepciones sobre las actitudes, éstas son esencialmente una medida de las preferencias y sentimientos expresados hacia un objeto, en este caso, la Ciencia y lo científico (Osborne et al. 2003). No obstante, esta conceptualización es demasiado amplia y aún no se ha alcanzado un consenso acerca de qué aspectos constituyen las actitudes hacia la Ciencia, por lo que los estudios anteriores han incorporado muchos componentes como la autoestima, la motivación hacia la Ciencia o el disfrute de la Ciencia, entre muchos otros (Osborne et al. 2003). 
Mientras que la actitud hacia la Ciencia fue un foco de investigación importante en Didáctica de las Ciencias, las teorías de la psicología social han influido en el estudio de las actitudes hacia la ciencia, por lo que en los últimos años el comportamiento, más que las actitudes en sí, se ha convertido en un foco de interés. Por lo tanto, muchos investigadores adoptaron modelos y teorías conductuales tales como la Teoría de la Conducta Planificada (Ajzen 1991) para comprender mejor la relación entre las actitudes y la conducta de los estudiantes. La teoría de Ajzen (1991) hace una clara distinción entre las actitudes hacia un objeto (es decir, actitudes hacia la ciencia) y las actitudes hacia una acción que debe realizarse con respecto al objeto (p. ej.: actitud hacia matricularse en una carrera en ciencias o actitud hacia participar en actividades científicas extracurriculares).

Por lo tanto, dado que las actitudes hacia la acción son mejores predictoras del comportamiento de las personas que las actitudes hacia el objeto mismo (Ajzen y Fishbein 1980), en este estudio nos enfocamos en examinar la actitud de los estudiantes hacia el estudio del contenido de la física o la biología (actitud hacia la acción a realizar sobre el objeto) en lugar de las actitudes de los estudiantes hacia el contenido de la física o la biología (actitud hacia el objeto). Este enfoque es consistente con modelos como el de la Teoría de Conducta Planeada (Ajzen 1991), y, por lo tanto, nos ayudará a examinar la interacción entre el género, el curso escolar y las actitudes hacia el estudio de la física y la biología.

\section{Método}

\section{Participantes y contexto}

Los datos de este estudio provienen de un extenso proyecto de investigación enfocado al desarrollo de un modelo de enseñanza STEM integrado para la etapa de Educación Primaria, por lo que la muestra está compuesta por 733 estudiantes $\left(M_{\text {edad }}=10.11, D E_{\text {edad }}=1.18 ; 46.9 \%\right.$ niñas) matriculados en el $3^{\text {er }}(n=65), 4^{\circ}(n=149), 5^{\circ}(n=232)$ y $6^{\circ}(n=287)$ curso de Educación Primaria. Los estudiantes provenían de un total de 29 Colegios de Educación Infantil y Primaria (CEIPs) situados en la provincia de Burgos (España), que han participado en un programa intensivo de enriquecimiento curricular de una semana de duración desarrollado en el Centro Rural de Innovación Educativa de Burgos (CRIEB).

\section{Instrumento y procedimiento}

Se ha empleado el instrumento School Science Attitudes Survey (SSAS) (Kennedy, Quinn y Taylor, 2016) en su versión en castellano (Toma y Meneses Villagrá en prensa) (Tabla 1). Se trata de un instrumento diseñado para medir las actitudes de los estudiantes de Educación Primaria hacia la asignatura de Ciencias de la Naturaleza en base a los seis constructos actitudinales (CA) más comunes en la literatura de actitudes hacia la Ciencia. Los CA (I) intenciones de matricularse en Ciencias de la Naturaleza, (II) disfrute de las clases de Ciencias de la Naturaleza, (III) autoeficacia en el aprendizaje de Ciencias de la Naturaleza, y (IV) dificultad percibida de las clases de Ciencias de la Naturaleza poseen una naturaleza unidimensional. Los dos constructos restantes, (V) utilidad y (VI) relevancia de las clases de Ciencias de la Naturaleza, poseen una naturaleza multidimensional. Más concretamente, el constructo 'utilidad de las clases de ciencias' está compuesto por dos dimensiones llamadas 'utilidad para las carreras científicas' y 'utilidad para la elección de otras carreras'. Por último, el constructo "relevancia de las clases de Ciencias de la Naturaleza" está formado a partir de las dimensiones 'relevancia para la sociedad' y 'relevancia para la vida personal'. Tanto los CA unidimensionales como las dimensiones subyacentes a los CA multidimensionales se miden a través de escalas de ítem único, bien de tipo Likert de cinco opciones (totalmente en desacuerdo- totalmente de acuerdo) o de diferencial semántico (p. ej.: divertido - aburrido). 
Esta escala ha sido previamente validada con estudiantes españoles de Educación Primaria, mostrando resultados que indican que la versión española del SSAS comparte una varianza satisfactoria con otros instrumentos destinados a medir las actitudes de los estudiantes hacia la Ciencia y su aprendizaje, confirmando así la validez convergente del SSAS en castellano. Se trata de un instrumento de reducida extensión y rápida administración, postulándose en su versión en castellano como un instrumento adecuado para medir las actitudes de los estudiantes hacia las clases de Ciencias (Toma y Meneses Villagrá en prensa). En este estudio, la confiabilidad del instrumento se ha medido empleando dos pruebas de consistencia interna. Así, el Alfa de Cronbach ha sido .763, resultados que se encuentran por encima del criterio mínimo de .70 estipulado para estudios exploratorios (Nunnally y Bernstein 1994) y que están en consonancia con recomendaciones relacionadas con el uso de escalas de autoinforme con pocos ítems y para muestras con estudiantes de poca edad (Newman y McNeil 1998). Además, las correlaciones ítem-total oscilaron entre .313 y .552, resultados asimismo superiores al criterio mínimo .30 (Nunnally y Bernstein 1994). Por tanto, en base a ambas pruebas, se puede concluir que la confiabilidad del instrumento empleado es satisfactoria.

El cuestionario empleado fue administrado en presencia del primer autor y en ausencia de los tutores convencionales de aula a la llegada de los estudiantes al CRIEB tras informar del carácter voluntario y anónimo de su participación.

Tabla 1. Constructos e ítems de la escala SSAS

\begin{tabular}{|c|c|}
\hline Constructo & Ítems \\
\hline (I) Intenciones & $\begin{array}{l}\text { 1. Es muy probable que me apunte a asignaturas de } \mathrm{CN} \text { al terminar } \\
\text { Educación Primaria (LT) }\end{array}$ \\
\hline (II) Disfrute & 2. Pienso que CN es: (SD aburrida - divertida) \\
\hline (III) Dificultad & 3. Me cuesta terminar las tareas para la clase de $\mathrm{CN}$ (LT) \\
\hline (IV) Autoeficacia & 4. Pienso que soy muy bueno en $\mathrm{CN}$ (LT) \\
\hline \multicolumn{2}{|l|}{ (V) Utilidad } \\
\hline Utilidad para estudios de ciencias & 5. Un trabajo como científico sería interesante (LT) \\
\hline Utilidad para estudios personales & $\begin{array}{l}\text { 6. Para mis futuros estudios, el conocimiento de las clases de } \mathrm{CN} \\
\text { será: (SD inútil - útil) }\end{array}$ \\
\hline \multicolumn{2}{|l|}{ (VI) Relevancia } \\
\hline Relevancia social & 7. La ciencia ayuda a mejorar la vida (LT) \\
\hline \multirow[t]{3}{*}{ Relevancia personal } & 8. Quiero aprender sobre las plantas de mi entorno (LT) \\
\hline & $\begin{array}{l}\text { 9. Para mi vida cotidiana, creo que } \mathrm{CN} \text { es (SD poco importante - } \\
\text { muy importante) }\end{array}$ \\
\hline & $\begin{array}{l}\text { 10. Quiero aprender sobre la electricidad y saber cómo se usa en } \\
\text { una casa (LT) }\end{array}$ \\
\hline
\end{tabular}

Notas: CN: Ciencias de la Naturaleza; LT: ítems de tipo Likert de 5 opciones (totalmente en desacuerdo - totalmente de acuerdo); SD: ítems de tipo diferencial semántico de 5 opciones de respuesta.

\section{Análisis de datos}

El interés por contenidos de biología y de física se ha determinado a partir del análisis de los ítems 8. Quiero aprender sobre las plantas de mi entorno (biología) y 10. Quiero aprender sobre la electricidady saber cómo se usa en una casa (física) de la dimensión 'relevancia para la vida personal'.

En relación con la primera pregunta de investigación, se empleó el estadístico $t$ de Student para muestras independiente para comparar el interés de las niñas y los niños por contenidos de física y de biología y varias ANOVAs univariantes para comparar el interés por contenidos de física y de biología en función de la edad. Se empleó una corrección de Bonferroni estableciéndose un valor de significancia más estricto $(p<.01)$ y así evitar errores de tipo I. 
Para la segunda pregunta de investigación, se empleó un método de análisis cuantitativo basado en técnicas de agrupamiento llamado clúster o de conglomerados. El análisis clúster es una técnica analítica centrada en sujetos que proporciona una manera de examinar cómo se combinan las variables dentro de los individuos (Everitt, Landau, Leese y Stahl 2011). Por lo tanto, el análisis clúster tiene por objeto la clasificación de los sujetos en diferentes grupos que posteriormente son analizados en búsqueda de características similares y distintivas que diferencia a cada subgrupo. En la literatura, se ha comprobado la utilidad del análisis clúster en su aplicación para la identificación de subgrupos significativos en contextos académicos (Battaglia, Di Paola y Fazio 2017; Fazio, Battaglia y Di Paola 2013; Paciello, Ghezzi, Tramontano, Barbaranelli y Fida 2016). Las técnicas de análisis clúster pueden ser divididas en dos grupos: jerárquico y no jerárquico (Everitt et al. 2011). En este estudio, se empleó el análisis clúster bietápico con medida de distancia logarítmica de verosimilitud y criterio de información bayesiano (BIC) (Schwarz 1978) para la determinación del número de clústeres a extraer. Posteriormente se empleó un método de clúster no jerárquico, llamado K-Medias para la extracción de los conglomerados que han determinado los perfiles de interés por contenidos de física o de biología de cada estudiante. Asimismo, se realizaron dos pruebas de independencia estadística Chi-cuadrado para examinar si los estudiantes estaban uniformemente representados en los perfiles en función del nivel escolar y el sexo y así determinar si las preferencias de las niñas y de los niños de Educación Primaria simulan patrones de matriculaciones de etapas secundarias y terciarias.

Para la tercera y última pregunta de investigación, se efectuaron una serie de pruebas de MANOVA, ANOVA y $t$ de Student con corrección de Bonferroni para analizar las diferencias entre los cuatro perfiles de interés en términos de actitudes hacia la asignatura Ciencias de la Naturaleza. Más específicamente, se examinaron las diferencias entre cada subgrupo en términos de cinco constructos actitudinales (CA): (I) intenciones de matricularse en ciencias, (II) disfrute durante las clases de Ciencias de la Naturaleza, (III) dificultad de las clases de Ciencias de la Naturaleza, (IV) percepción de autoeficacia en Ciencias de la Naturaleza, y (V) utilidad percibida de las clases de Ciencias de la Naturaleza.

\section{Resultados}

\section{P1. ¿Cómo afecta el sexo y el curso escolar a las preferencias por estudiar contenidos científicos de física y de biología?}

La prueba $t$ para muestras independientes señaló que no hay diferencias significativas en el interés por contenidos de biología entre niñas $(M=3.23$, $S D=1.12)$ y niños $(M=3.36, S D=$ 1.21). Sin embargo, los niños reportaron un interés significativamente mayor por contenidos de física que las niñas, $t(731)=-5.33, p<.001$. Estos resultados aparecen en la figura 1 .

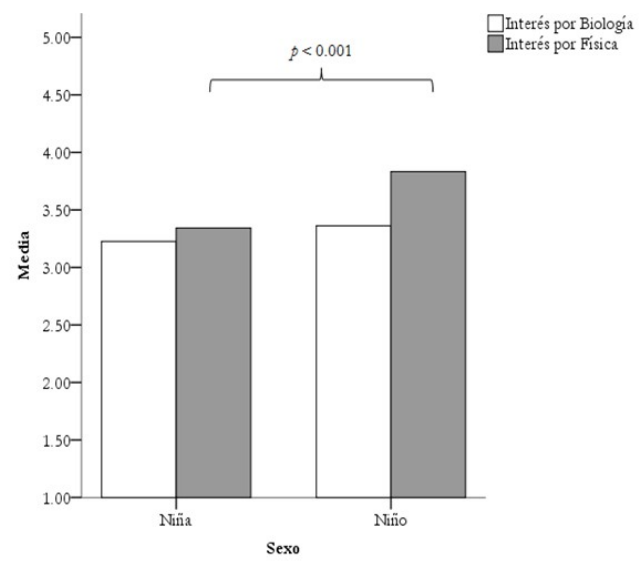

Figura 1. Comparaciones según sexo para interés en biología o física 
$\mathrm{Al}$ controlar la variable sexo, el ANOVA univariante indicó que las niñas matriculadas en el $6^{\circ}$ curso de Educación Primaria poseen un interés significativamente menor por el contenido de biología en comparación con las niñas matriculadas en el tercer curso, $F(3,340)=3.9, p=$. 009. También se ha identificado un descenso en el interés de las niñas por contenidos de física a medida que aumenta el curso escolar, sin embargo, los resultados no alcanzaron significancia estadística. En cuanto a los niños, se ha identificado que a medida que aumenta el curso escolar, su interés por contenidos de biología disminuye y su interés por contenidos de física aumenta, aunque ninguno de los dos resultados es significativo. Estos resultados aparecen en la figura 2.

(I)

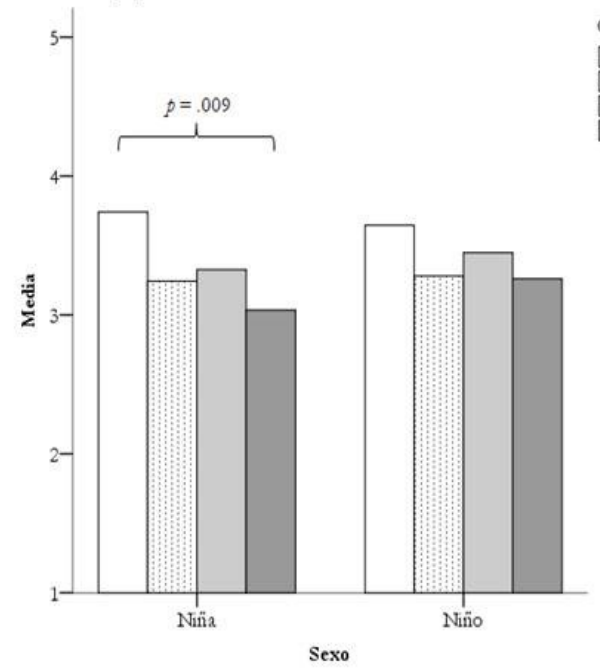

(II)

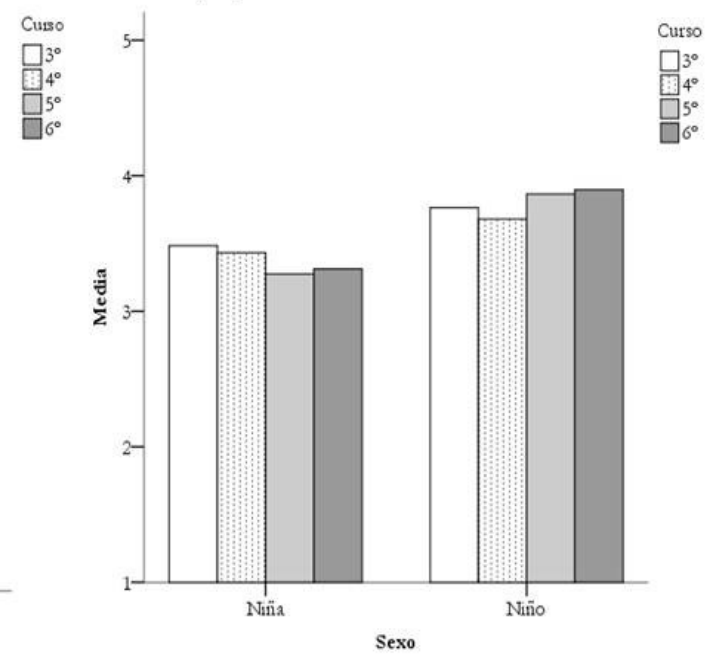

Figura 2. Comparaciones según nivel escolar para interés en (I) biología y (II) física

\section{P2. Las preferencias, ¿simulan patrones de matriculaciones identificadas en etapas secundarias y terciarias?}

La prueba clúster bietápica reveló la existencia de siete clústeres con medida Silhouette de cohesión y separación adecuada (.5). No obstante, dado que los clústeres formados con pocos estudiantes pueden resultar poco relevantes desde un punto de vista pedagógico (Battaglia et al. 2017), se realizaron sucesivos análisis clúster de tipo bietápico especificando un menor número de clústeres a extraer, obteniéndose que una solución de cuatro subgrupos posee la medida de Silhouete más adecuada (.6), y una estructura de conglomerados razonable (Struyf, Hubert y Rousseeuw 1997). Determinado el número de subgrupos a extraer, se efectuó un análisis K-Medias para extraer perfiles en función del interés por contenidos de física o de biología.

El análisis clúster K-Medias reveló una solución de cuatro clústeres (iteración = 3). El MANOVA reveló diferencias significativas entre los cuatro clústeres en ambos ítems, Wilks Lamb$\mathrm{da}=.100, F(6,1456)=525.362, p<.001, \eta^{2}=.684$. Dos pruebas ANOVA mostraron que el efecto principal de la pertenencia a los subgrupos fue igualmente significativo tanto por la preferencia de contenidos de biología $F(3,729)=401.8, p<.001$, como por física $F(3,729)=$ 746.9, $p<.001$. Las comparaciones post-hoc de Bonferroni revelaron la existencia los cuatro siguientes perfiles (Figura 3): 
i. «Interés por física», con 72 estudiantes $\left(M_{\text {edad }}=10.28 ; D E_{\text {edad }}=1.10\right)$ que mostraron un interés significativamente mayor por contenidos de física que por contenidos de biología.

ii. «Interés por biología», con 221 estudiantes $\left(M_{\text {edad }}=10.02 ; D E_{\text {edad }}=1.20\right)$ con un interés por contenidos de biología significativamente mayor que por los propios de física.

iii. «Bajo interés general», con 85 estudiantes $\left(M_{\text {edad }}=10.29 ; D E_{\text {edad }}=1.10\right)$ que reportaron un bajo interés tanto por contenidos de física como de biología.

iv. «Alto interés general», con 355 estudiantes $\left(M_{\text {edad }}=10.10 ; D E_{\text {edad }}=1.21\right)$ que mostraron un alto interés por ambos contenidos de física y de biología.

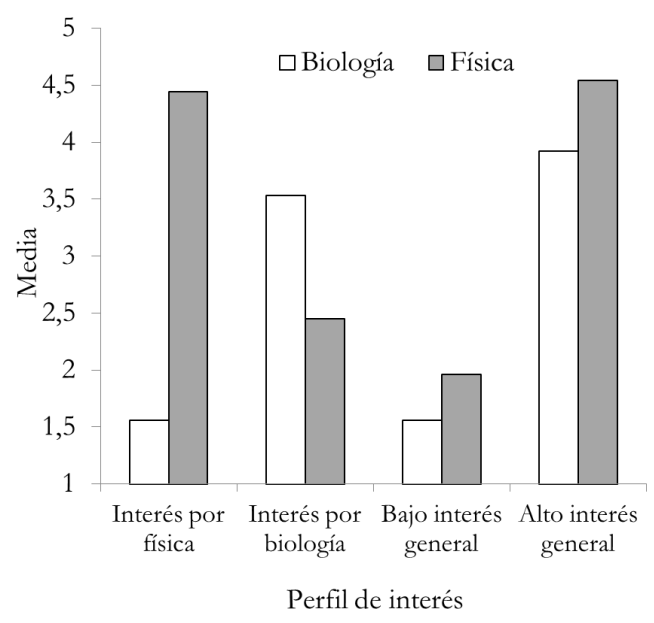

Figura 3. Perfiles según el interés por contenidos de física o de biología

En relación con el nivel escolar, la prueba de independencia estadística Chi-cuadrado reveló que los participantes estaban uniformemente representados en los cuatro perfiles, $\chi^{2}(9, n=$ $733)=5.125, p=.823, \mathrm{phi}=.82$. Sin embargo, con respecto al sexo de los estudiantes, se encontraron diferencias significativas en la distribución de estos, $\chi^{2}(3, n=733)=20.843, p<$. 001 , phi $=.17$. Así, se encontró una significativa sobrerrepresentación de las niñas (126 de $221,57 \%$ ) con respecto a los niños (95 de 221, 43\%) en el perfil «interés por biología», y una significativa infrarrepresentación de las niñas (28 de 72, 38.9\%) en el perfil «interés por física» con respecto de los niños (44 de 72, 61.1\%). Además, hubo significativamente más niñas (48 de $85,56.5 \%$ ) que niños ( 37 de $85,43.5 \%$ ) agrupadas en el perfil «bajo interés general», y significativamente menos niñas (142 de 355, 40\%) que niños (213 de 355, 60\%) en el perfil «alto interés general». La figura 4 muestra la distribución de los estudiantes según sexo en cada perfil de preferencia.

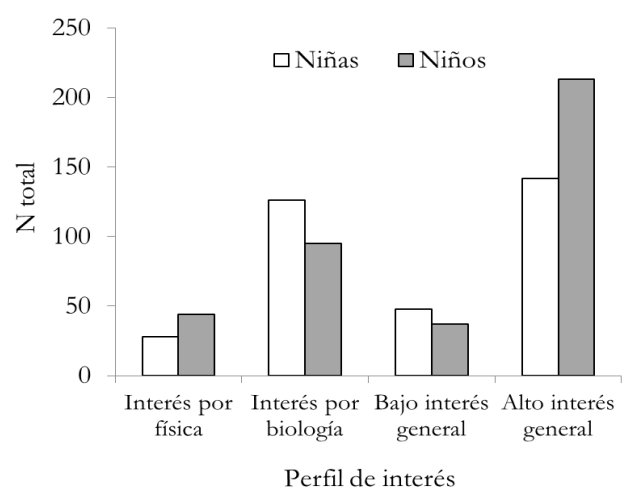

Figura 4. Distribución de los estudiantes en los perfiles derivados según sexo 
P3. ¿Existen diferencias en las actitudes de los estudiantes hacia la asignatura de Ciencias de la Naturaleza en función de su preferencia por contenidos de física o de biología?

El MANOVA reveló diferencias significativas en los CA entre los cuatro perfiles de interés, Wilks Lambda $=.837, \mathrm{~F}\left(1^{2}, 1921\right)=11.142, \mathrm{p}<.001, \eta^{2}=.058$. Cinco ANOVAs univariantes con corrección de Bonferroni revelaron diferencias significativas para cuatro de los cinco CA: «intención de matricularse en Ciencias de la Naturaleza» F $(3,729)=29.829, p<.001, \eta^{2}=$. 62, «disfrute durante las clases de Ciencias de la Naturaleza» $F(3,729)=30.768, p<.001, \eta^{2}$ $=.62$, «percepción de autoeficacia en Ciencias de la Naturaleza» $F(3,729)=7.702, p<.001$, $\eta^{2}=.75$, y «utilidad de las clases de Ciencias de la Naturaleza» $F(3,729)=145.204, p<.001$, $\eta^{2}=.16$

Las comparaciones post-hoc de Bonferroni reportaron que los estudiantes del grupo 'alto interés general' son los que poseen significativamente más intenciones de seguir estudios de ciencias y los que han manifestado significativamente mayor disfrute durante las clases de ciencias, especialmente en comparación con los estudiantes del perfil «bajo interés general»e «interés en física». Asimismo, los estudiantes del perfil «alto interés general» mostraron significativamente mayor autoeficacia en ciencias que los otros tres grupos, siendo los estudiantes con 'bajo interés general' los que reportaron menor autoeficacia de todos los grupos. Por último, los estudiantes del perfil «alto interés general» asimismo concibieron las clases de Ciencias de la Naturaleza como significativamente más útiles que los demás perfiles. Estos resultados se recogen en la figura 5.

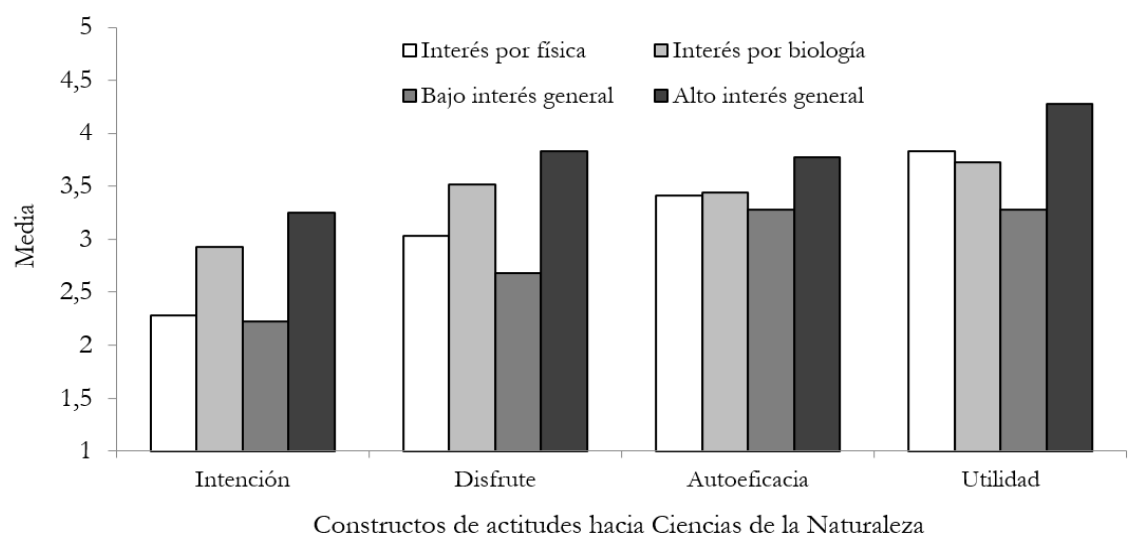

Figura 5. Comparaciones entre actitudes hacia Ciencias de la Naturaleza según el interés por contenidos de biología o de física

\section{Interpretación y discusión}

En conjunto, los resultados de este estudio confirman las premisas de inicio. Así, se han identificado al menos cuatro aspectos que resultan de gran preocupación para la educación científica en España.

En primer lugar, se ha identificado un perfil actitudinal caracterizado por un alto interés por contenidos de biología en el que predominaban las niñas y un perfil que se caracteriza por un alto interés por física, representado mayoritariamente por niños. Estos resultados resultan preocupantes porque evidencian que ya desde etapas elementales del sistema educativo se pueden identificar patrones de interés en ciencias muy similares a los registrados por los datos nacionales de matriculación en estudios universitarios (MECD 2016). De este modo, las niñas 
tienen más interés por contenidos de biología y menos por los propios de física, sucediendo lo contrario en el caso de los niños, siendo ésta una diferencia que se plasma en las estadísticas de matriculaciones en estudios terciarios pero que no ha sido previamente estudiada a nivel de Educación Primaria.

En segundo lugar, se ha observado que las niñas son mayoría en un perfil caracterizado por un bajo interés general, y son minoría en el perfil con un alto interés tanto por contenidos de biología como de física. Estos datos son asimismo preocupantes ya que coinciden con los resultados registrados en Educación Secundaria a nivel nacional (de Pro y Pérez 2014; Vázquez y Manassero 2008) e internacional (Caleon y Subramaniam 2008; Denessen et al. 2015; DeWitt y Archer 2015; George 2006; Hacieminoglu 2016) y que muestran una tendencia menor de las niñas por la Ciencia que los niños. Si bien los estudios mencionados han sido desarrollados principalmente con estudiantes de Educación Secundaria, el presente estudio evidencia que este patrón comienza desde la etapa de Educación Primaria.

En tercer lugar, se ha identificado un descenso en el interés de las niñas tanto por contenidos de física como de biología a medida que aumenta el nivel escolar, lo que coincide con estudios anteriores que señalan que las niñas pierden más rápidamente el interés por la Ciencia en cursos superiores en comparación con los niños (Marginson, Tytler, Freeman y Roberts 2013). Estos datos resultan preocupantes debido a que las aspiraciones profesionales podrían estar mayormente formadas a la edad de 13 años, especialmente en las niñas (Lindahl 2007), siendo cada vez más difícil involucrarlas en disciplinas científicas en niveles posteriores.

Por último, al examinar las actitudes de los estudiantes hacia la asignatura de Ciencias de la Naturaleza en función del perfil de interés, se ha observado que aquellos estudiantes con mejores actitudes poseen asimismo un alto interés general tanto por contenidos de física como de biología. La mayoría de las niñas de esta muestra han sido categorizadas dentro del perfil «alto interés general», seguido por «interés en biología», por lo que se podría concluir que en Educación Primaria las actitudes aún son positivas, aunque ya se advierten diferencias de género en cuanto al interés y las preferencias y un descenso en las actitudes, especialmente en las intenciones de seguir estudios de ciencias, que ha sido el constructo actitudinal peor valorado, seguido por el disfrute de las clases de ciencias, resultados coincidentes con investigaciones previas (Denessen et al. 2015).

\section{Implicaciones educativas y de investigación}

Los resultados de este estudio subrayan la necesidad de diseñar y desarrollar intervenciones educativas desde edades tempranas para despertar el interés y retener a las niñas en la Ciencia, especialmente en aquellas disciplinas en las que se encuentran más infrarrepresentadas, como la física. Estas intervenciones deberían realizarse antes de la etapa de Educación Secundaria, tal y como se viene resaltando en la literatura (Marbá-Tallada y Márquez Bargalló 2010) y además debido a que, tal y como muestran los resultados de este estudio, el interés por la física y la biología parecería forjarse desde la Educación Primaria. Las intervenciones educativas deben abordar al menos dos aspectos esenciales: el uso de metodologías activas para la enseñanza y el aprendizaje de las ciencias, y la reducción de sesgo de género en el rendimiento académico en ciencias.

En cuanto al uso de metodologías activas, la indagación escolar o el aprendizaje basado en problemas han mostrado ser efectivas en aumentar la participación de los estudiantes durante las clases y en mejorar la percepción de utilidad de las ciencias y el disfrute durante las clases de ciencias (Demirel y Dagyar 2016; Romero-Ariza 2017; Schroeder, Scott, Tolson, Huang y Lee 2007; Toma y Greca 2018). Dado que las diferencias entre los niños y las niñas se atribuye a factores de aversión al riesgo y a la competitividad, favoreciendo a los niños (Booth, 
Cardona-Sosac y Nolen, 2014; Niederle y Vesterlund 2010) y a que las niñas tienden a subestimar sus habilidades en la elección de trayectorias profesionales (Rapoport y Thibout 2018), las metodologías de enseñanza y aprendizaje activas podrían ser efectivas para reducir el nivel de competitividad durante las clases de ciencias estructurando grupos de trabajos que fomenten la participación colaborativa y la búsqueda conjunta de perspectivas y de resolución de problemas (Gillies 2003).

En relación con la existencia de sesgos de género, parecería que las expectativas de los padres y de los maestros en cuanto a las competencias de los niños y las niñas posee sesgos de género y podrían influir negativamente en las actitudes y el rendimiento de las niñas, disminuyendo su autoconcepto (Gunderson et al. 2012). Además, se ha comprobado que los maestros evalúan las capacidades de las niñas como inferiores a las de los niños (Dickhauser y Meyer 2006). Si bien estos resultados están más relacionados con las matemáticas, existen evidencias que indican la existencia de asociaciones estereotipadas que vinculan a los hombres con carreras profesionales en ciencia y a las mujeres con las artes y la familia (Nosek, Banaji y Greenwald 2002), así como a las mujeres menos competentes en ciencias que los hombres (Eagly y Mladinic 1993).

Por último, en este estudio se ha identificado que el interés por contenidos de física o de biología comienza a establecerse a partir de la etapa de Educación Primaria. Futuros estudios deberían abordar estos resultados para examinar qué factores intervienen para que desde una edad tan temprana pueda haber una preferencia tan marcada y diferenciada según el género de los estudiantes.

\section{Conclusiones}

La presente investigación busca entender mejor a partir de qué edad se produce una segregación horizontal de las mujeres en las disciplinas científicas mediante el análisis de las preferencias de 344 niñas y 389 niños de Educación Primaria por contenidos de física o de biología. Además, se ha examinado las actitudes hacia las clases de Ciencias de la Naturaleza en función de las preferencias por unos u otros contenidos. El análisis de conglomerados reveló la existencia de un perfil actitudinal sesgado sexualmente, caracterizado por un alto interés por el contenido de las ciencias biológicas y un bajo interés por el aprendizaje de la física por parte de las niñas, y resultados opuestos en el caso de los niños. Además, en comparación con los niños, las niñas han sido mayoría en los perfiles actitudinales caracterizados por un bajo interés por la física y la biología, y han sido minoría en los perfiles caracterizados por un alto interés por ambas disciplinas.

El análisis de los constructos actitudinales de los miembros pertenecientes a los diferentes perfiles ilustraron que el grupo de los estudiantes con un alto interés general por los contenidos de física y de biología reportaron mejores actitudes hacia la ciencia escolar que el resto de los grupos, lo que sitúa a las niñas con este tipo de perfil en riesgo de exclusión educativa en ciencias, ya desde la Educación Primaria.

En conjunto, estos resultados señalan que las trayectorias académicas y profesionales de los estudiantes, así como su interés por disciplinas científicas de física o de biología, se empiezan a conformar desde niveles elementales del sistema educativo, suceso que podría dar lugar al posterior descenso de mujeres matriculadas en carreras universitarias de ciencias. La importancia de estos resultados reside en subrayar la necesidad de desarrollar intervenciones educativas desde la etapa de Educación Primaria para reducir las diferencias de género detectadas sobre actitudes hacia la ciencia escolar e interés por disciplinas científicas. 


\section{Agradecimientos}

Este estudio fue financiado por la edición 2017-2021 del programa de contratos de investigación predoctorales de la Universidad de Burgos y parcialmente financiado por el proyecto MINECO-AEI EDU 2017-89405-R.

Extendemos nuestro agradecimiento de manera especial al Centro Rural de Innovación Educativa de Burgos (CRIEB) y a todos los centros educativos que han formado parte de este estudio.

\section{Referencias}

AAAS. (1993) Benchmarks for scientific literacy. Washington: American Association for the Advancement of Science.

AGARD. (1996) Advisory Report 330: Anthropomorphic dummies for crash and escape system testing. Quebec: Canada Communication Group.

Ajzen, I. (1991) The theory of planned behavior. Organizational Behavior and Human Decision Processes, 50(2), 179-211.

Ajzen, I., Fishbein, M. (1980) Understanding attitudes and predicting social behavior. New Jersey: Prentice-Hill.

Battaglia, O. R., Di Paola, B., Fazio, C. (2017) K-means clustering to study how student reasoning lines can be modified by a learning activity based on Feynman's unifying approach. Eurasia Journal of Mathematics, Science and Technology Education, 13(6), 2005-2038. doi:10.12973/eurasia.2017.01211a

Beede, D. N., Julian, T. A., Langdon, D., McKittrick, G., Khan, B., Doms, M. E. (2011) Women in STEM: A gender gap to innovation. Economics and Statistics Administration, 4 11. doi:10.2139/ssrn.1964782

Berkhout, E., Sattinger, M., Theeuwes, J., Volkerink, M. (2012) Into the gap. Exploring skills and mismatches. Recuperado de: https://research.randstad.es/wpcontent/uploads/2015/12/into-the-gap.pdf

Blickenstaff, J. C. (2005) Women and science careers: Leaky pipeline or gender filter? Gender and Education, 17(4), 369-386. doi:10.1080/09540250500145072

Booth, A., Cardona-Sosac, L., Nolen, P. (2014) Gender differences in risk aversion: Do singlesex environments affect their development. Journal of Economic Behavior and Organization, 99, 126-154.

Bug, A. (2003) Has feminism changed physics. Signs, 28(3), 881-899. doi:00979740/2003/2803-0008

Buser, T., Niederle, M., Oosterbeek, H. (2014) Gender, competitiveness, and career choices. The Quarterly Journal of Economics, 129, 1409-1447.

Caleon, I. S., Subramaniam, R. (2008) Attitudes towards science of intellectually gifted and mainstream upper primary students in Singapore. Journal of Research in Science Teaching, 45(8), 940-954. doi:10.1002/tea.20250

Collins, C., Kenway, K., McLeod, J. (2000) Factors influencing the educational performance of males and females in school and their initial destinations after leaving school. Recuperado de

http://siandvasupport.sydneyinstitute.wikispaces.net/file/view/Factors+influsencing+th $\mathrm{e}+$ performance + of + males + and + females + in + school.pdf

Conkey, M. W. (2003) Has feminism changed archaeology? Signs, 28(3), 867-880. 
Dasgupta, N. (2011) Ingroup experts and peers as social vaccines who inoculate the selfconcept: The stereotype inoculation model. Psychological Inquiry, 22(3), 231-246.

de Pro Bueno, A., Pérez Manzano, A. (2014) Actitudes de los alumnos de Primaria y Secundaria ante la visión dicotómica de la Ciencia. Enseñanza de Las Ciencias, 32(3), 111132. doi: $10.5565 / \mathrm{rev} /$ ensciencias. 1015

Deloitte. (2016) Women in STEM. Technology, career pathways and the gender pay gap. London: Deloitte.

Demirel, M., Dagyar, M. (2016) Effects of problem-based learning on attitude: A metaanalysis study. Eurasia Journal of Mathematics, Science and Technology Education, 12(8), 21152137. doi:10.12973/eurasia.2016.1293a

Denessen, E., Vos, N., Hasselman, F., Louws, M. (2015) The relationship between primary school teacher and student attitudes towards Science and Technology. Education Research International, 1-7. doi:10.1155/2015/534690

DeWitt, J., Archer, L. (2015) Who aspires to a science career? A comparison of survey responses from primary and secondary school students. International Journal of Science Education, 37(13), 2170-2192. doi:09500693.2015.1071899

Dickhauser, O., Meyer, U. (2006) Gender difference in young children's math ability attributions. Psychological Science, 48(1), 3-16.

Eagly, A. H., Mladinic, A. (1993) Are people prejudiced against women? Some answers from research on attitudes, gender stereotypes, and judgments of competence. European Review of Social Psychology, 5(1), 1-35.

EC. (2004) Europe needs more scientists: Report by the high-level group on increasing human resources for Science and Technology. Brussels: European Commission.

EC. (2013) Special eurobarometer 401: Responsible Research and Innovation (RRI), Science and Technology. Brussels: European Commission.

Everitt, B. S., Landau, S., Leese, M., Stahl, D. (2011) Cluster Analysis. Chichester: John Wiley \& Sons, Ltd.

Fazio, C., Battaglia, O. R., Di Paola, B. (2013) Investigating the quality of mental models deployed by undergraduate engineering students in creating explanations: the case of thermally activated phenomena. Physical Review Physics Education Research, 9(2), 1-21.

Gardner, P. L. (1975) Attitudes to science: A Review. Studies in Science Education, 2, 1-41.

George, R. (2006) A cross-domain analysis of change in students' attitudes toward science and attitudes about the utility of science. International Journal of Science Education, 28(6), 571589. doi:10.1080/09500690500338755

Gillies, R. M. (2003) Structuring cooperative group work in classrooms. International Journal of Educational Research, 39(1-2), 35-49. doi:10.1016/S0883-0355(03)00072-7

Gunderson, E. A., Ramirez, G., Levine, S. C., Beilock, S. L. (2012) The role of parents and teachers in the development of gender-related math attitudes. Sex Roles, 66(3-4), 153166. doi:10.1007/s11199-011-9996-2

Hacieminoglu, E. (2016) Elementary school students' attitude toward science and related variables. International Journal of Environmental and Science Education, 11(2), 35-52. doi:10.12973/ijese.2016.288a 
Heybach, J., Pickup, A. (2017) Whose STEM? Disrupting the gender crisis within STEM. Educational Studies, 53(6), 614-627. doi:10.1080/00131946.2017.1369085

INE. (2017) Mujeres y hombres en España. Educación. Recuperado de https://goo.gl/RC2UKd

Kennedy, J., Quinn, F., Taylor, N. (2016) The school science attitude survey: A new instrument for measuring attitudes towards school science. International Journal of Research y Method in Education, 39(4), 422-445. doi:10.1080/1743727X.2016.1160046

Lindahl, B. (2007) Longitudinal study of students' attitudes towards science and choice of career. En proceedings de $80^{\text {th }}$ session of the International Conference of the National Association for Research in Science Teaching. New Orleans.

Ley Orgánica para la Mejora de la Calidad Educativa (LOMCE) (Ley Orgánica 8/2013, 9 de diciembre). Boletín Oficial del Estado, núm. 295, 2013, 10 de diciembre. Referencia: BOE-A-2013-12886.

Marbá-Tallada, A., Márquez Bargalló, C. (2010) ¿Qué opinan los estudiantes de las clases de ciencias? Un estudio transversal de sexto de primaria a cuarto de ESO. Enseñanza de las Ciencias, 28(1), 19-30.

Marginson, S., Tytler, R., Freeman, B., Roberts, K. (2013) STEM: Country comparisons. International comparisons of science, technology, engineering and mathematics (STEM) education. Melbourne: Australian Council of Learned Academies.

MECD. (2016) Datos y cifras del sistema universitario español. Curso 2015/2016. Secretaría General Técnica.

Morrell, C., Parker. C. (2015) Solving the education equation: A new model for improving STEM work.force outcomes through academic equity. Recuperado de: www.napequity.org/solvingeducation-equation

Newman, I., McNeil, K. (1998) Conducting survey research in the social sciences. New York: University Press of America.

Niederle, M., Vesterlund, L. (2010) Explaining the gender gap in math test scores: The role of competition. Journal of Economic Perspectives, 24(2), 129-144. doi:10.1257/jep.24.2.129

Nosek, B. A., Banaji, M. R., Greenwald, A. G. (2002) Harvesting implicit group attitudes and beliefs from a demonstration website. Group Dynamics: Theory, Research, and Practice, 6(1), 101-115.

NRC. (2012) A framework for K-12 science education: Practices, crosscutting concepts, and core ideas. Washington: The National Academies Press.

NSF. (2013) Women, minorities, and persons with disabilities in Science and Engineering. Washington: National Science Foundation.

Nunnally, J. C., Bernstein, I. H. (1994) Psychometric theory. New York: McGraw-Hill.

OECD. (2013) Education at a glance 2013: OECD indicators. Paris: OECD Publishing.

OECD., UNESCO. (2003) Literacy skills for the world of tomorrow - Further results from PIS A 2000. Paris: OECD.

Osborne, J., Dillon, J. (2008) Science education in Europa. Critical reflections. London: Nuffield Foundation. 
Osborne, J., Simon, S., Collins, S. (2003) Attitudes towards science: A review of the literature and its implications. International Journal of Science Education, 25(9), 1049-1079. doi:10.1080/0950069032000032199

Paciello, M., Ghezzi, V., Tramontano, C., Barbaranelli, C., Fida, R. (2016) Self-efficacy configurations and wellbeing in the academic context: A person-centered approach. Personality and Individual Differences, 99, 16-21. doi:10.1016/j.paid.2016.04.083

Ramsden, J. M. (1998) Mission impossible? Can anything be done about attitudes to science? Interntional Journal of Science Education, 20, 125-137.

Rapoport, B., Thibout, C. (2018) Why do boys and girls make different educational choices? The influence of expected earnings and test scores. Economics of Education Review, 62, 205-229. doi:10.1016/j.econedurev.2017.09.006

Romero-Ariza, M. (2017) El aprendizaje por indagación: ¿existen suficientes evidencias sobres sus beneficios en la enseñanza de las ciencias? Revista Eureka sobre Enseñanza y Divulgación de las Ciencias, 14(2), 286-299.

Schiebinger, L. (2003) Introduction: Feminism inside the sciences. Signs, 28(3), 859-866.

Schiebinger, L., Klinge, I., Sánchez de Madariaga, I., Paik, H. Y., Schraudner, M., Stefanick, M. (2011) Gendered innovations in science, Health y medicine, engineering and environment. Recuperado de http://genderedinnovations.stanford.edu/index.html

Schroeder, C. M., Scott, T. P., Tolson, H., Huang, T.-Y., Lee, Y.-H. (2007) A Meta-Analysis of national research: Effects of teaching strategies on student achievement in science in the United States. Journal of Research in Science Teaching, 44(10), 1436-1460. doi:10.1002/tea

Schwarz, G. E. (1978) Estimating the dimension of a model. Annals of Statistics, 6(2), 461-464.

Sikora, J., Pokropek, A. (2012) Gender segregation of adolescent science career plans in 50 countries. Science Education, 96(2), 234-264. doi:10.1002/sce.20479

Struyf, A., Hubert, M., Rousseeuw, P. J. (1997) Integrating robust clustering techniques in SPLUS. Computational Statistics \& Data Analysis, 26, 17-37. doi:10.1016/S01679473(97)00020-0

Toma, R. B., Greca, I. M. (2018) The effect of integrative STEM instruction on elementary students' attitudes toward Science. EURASLA Journal of Mathematics, Science and Technology Education, 14(4), 1383-1395. doi:10.29333/ejmste/83676

Toma, R. B., Meneses Villagrá, J. A. (en prensa) Validation of the Spanish single-item School Science Attitude Survey (S-SSAS) for elementary education. PLOS ONE.

UNESCO. (2017) Cracking the code: Girls' and women's education in science, technology, engineering and mathematics (STEM). París: United Nations Educational, Scientific and Cultural Organization. Recuperado de: http://unesdoc.unesco.org/images/0025/002534/253479e.pdf

Vázquez, A., Manassero, M. A. (2008) El declive de las actitudes hacia la ciencia de los estudiantes: un indicador inquietante para la educación científica. Revista Eureka sobre Enseñanza y Divulgación de las Ciencias, 5(3), 274-292.

Weiss, H., Songer, T., Fabio, A. (2001) Fetal deaths related to maternal injury. Journal of the American Medical Association, 15, 1863-1868. 